\title{
Vibration Control of the Boom System of Truck-Mounted Concrete Pump Based on Constant-Position Commandless Input Shaping Technique
}

\author{
Rongsheng Liu, Yingjie Gao, Sen Yang, and Yulin Yang \\ College of Mechanical Engineering, Yanshan University, 438 Hebei Avenue, Qinhuangdao 066004, China \\ Correspondence should be addressed to Rongsheng Liu; rsliu@ysu.edu.cn
}

Received 13 April 2015; Revised 23 June 2015; Accepted 23 June 2015

Academic Editor: Mohammad Elahinia

Copyright (C) 2015 Rongsheng Liu et al. This is an open access article distributed under the Creative Commons Attribution License, which permits unrestricted use, distribution, and reproduction in any medium, provided the original work is properly cited.

\begin{abstract}
Vibration of the boom system is inevitably caused by periodic disturbances, which are induced by pumping concrete. In this paper, an active control strategy of constant-position commandless input shaping technique is developed to suppress vibration. Based on a set of independent modal equations obtained by adopting modal approach, the double-impulse control with reverse direction is proposed, which is aiming at not only suppressing vibration, but also avoiding the variation of the equilibrium position of the boom system after active control action. And the characteristic of time-lag existing in real system is also taken into account for optimizing the control action. Experiments of vibration control were implemented on a 52-meter-long five-boom system to verify the proposed control strategy.
\end{abstract}

\section{Introduction}

The truck-mounted concrete pump is a kind of special machinery for conveying and pouring concrete, and it becomes essential in various construction sites. The boom system of truck-mounted concrete pump is a typical flexible multibody dynamics system, so that serious vibration of the boom system is inevitably caused by pumping concrete and large motion. Because of the vibration, the end of the boom system cannot reach the desired position, the waste of material increases greatly, and accidents arise frequently during construction. Furthermore, the dynamic stress induced by the vibration will reduce the fatigue life of the boom system seriously. However, the vibration of the boom system becomes more serious accompanied with the application of high pressure pump, large discharge pump, and long booms. Therefore, vibration control of the boom system becomes necessary.

In recent years, an increasing number of researches are devoted to vibration control of the boom system. In order to avoid the influence of vibration, a closed-loop detection and open-loop control strategy is proposed for trajectory tracking of single joint, in which the original trajectory is also optimized [1]. By considering the truck-mounted concrete pump as two subsystems, the boom and the concrete pump, Cazzulani et al. study the effects on boom vibration imposed by each subsystem, and the research achievement provides the theoretical basis for boom vibration control $[2,3]$. Furthermore, considering the vibration excited in two cases of large motion and concrete pumping, Resta et al. develop the independent modal control [4] and the active control strategy composed of a feedback control and a feed-forward control to suppress the booms vibration [5]. In [6], a disturbance estimator is proposed to compensate the disturbance by using the actuators which are used to drive booms. Huang et al. propose an active control strategy for boom vibration, which is composed of modal filter and optimal control theory [7]. In [8], a compensation control method is proposed for vibration control, which depends on vibratory trajectory prediction of the boom system.

According to the aforementioned literatures, the control methods are mainly proposed to deal with large motion and external disturbance. However, considering the vibration 
excited by pumping concrete is more common and serious in construction, and the periodic disturbances caused by pumping concrete are hardly eliminated, therefore, the research of vibration control aiming at external disturbance is emphasized in this paper.

Input shaping technique (IST) is an effective method for suppressing unnecessary residual vibration of flexible mechanism [9-12]. It acts as a command filter based on the impulse response of the system with the information of the vibration, and it has been proved to be more effective by many applications. To deal with the adverse effects of nonlinearity, time variation, and other characteristics, researchers develop IST to various advanced forms including adaptive input shaper, time-optimal input shaper, and robust input shaper [13-16], which enhance its adaptivity and robustness. Commandless input shaping technique (CIST) as a new form of IST is first proposed in the literature [17], which is aimed at suppressing vibration of the flexible structure caused by external disturbance. Differing from IST, the special impulse is induced according to the principle of CIST, and the system response of the impulse is able to counteract the original vibration completely [18]. However, the deviation of the equilibrium position of the boom always results from the impulse in practical application.

In this work, an active control strategy of constant-position CIST is developed, which is also based on independent modal approach. According to the decoupled modal equations, double-impulse control with reverse direction is designed for vibration control to satisfy the practical application and avoiding the variation of the equilibrium position after implementing control action. Finally, the proposed active control strategy is tested on a real 52-meter-long five-boom system.

\section{The Model of the Boom System}

In this work, the research object is a 52-meter-long five-boom system. As shown in Figure 1, the boom system is mainly composed of five booms and five hydraulic cylinders. Since the sectional dimension of each boom is smaller compared to its length, the booms are all considered as Euler-Bernoulli beam for analysis, and the axial and shear deformations of the booms are all neglected.

In this section, the coordinate system is established as Figure 2 shows. The origin of the $i$ th moving coordinate frame is attached to the initial point of the $i$ th boom, and the line between the initial point and final point is taken as $X_{i}$-axis.

In Figure 2, the $i$ th boom in the corresponding moving frame $O_{i} X_{i} Y_{i}$ is represented by vector $\mathbf{r}_{i}$, and $\mathbf{r}_{i}=\left[l_{i} 0\right]^{\mathrm{T}}$, in which $l_{i}$ represents the length of the boom. In this section, the subscript $i$ represents the number of the booms, and $i=$ $1, \ldots, 5 . \mathbf{p}_{i}$ represents the position vector of point $P$ attached to the $i$ th boom, and $\mathbf{p}_{i}=\mathbf{u}_{i}+\mathbf{v}_{i}$, in which $\mathbf{u}_{i}=\left[x_{i} 0\right]^{\mathrm{T}}$, and $\mathbf{v}_{i}=\left[\begin{array}{ll}0 & v_{i}\end{array}\right]^{\mathrm{T}} . \mathbf{v}_{i}$ represents the bending deformation at this point. Therefore, the position vector of point $P$ in inertial frame $O X Y$ can be expressed as

$$
\mathbf{p}_{i 0}=\sum_{k=1}^{i-1}\left(\mathbf{R}_{k} \mathbf{r}_{k}\right)+\mathbf{R}_{i} \mathbf{p}_{i}
$$

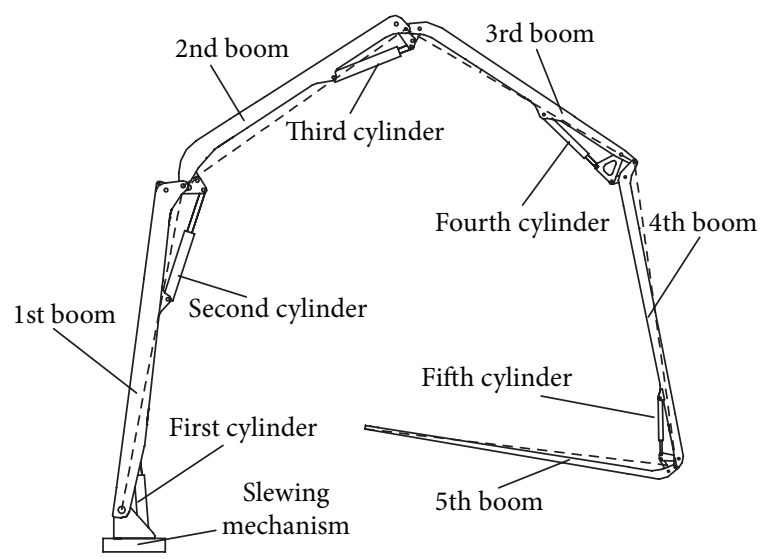

FIGURE 1: Structural drawing of the boom system.

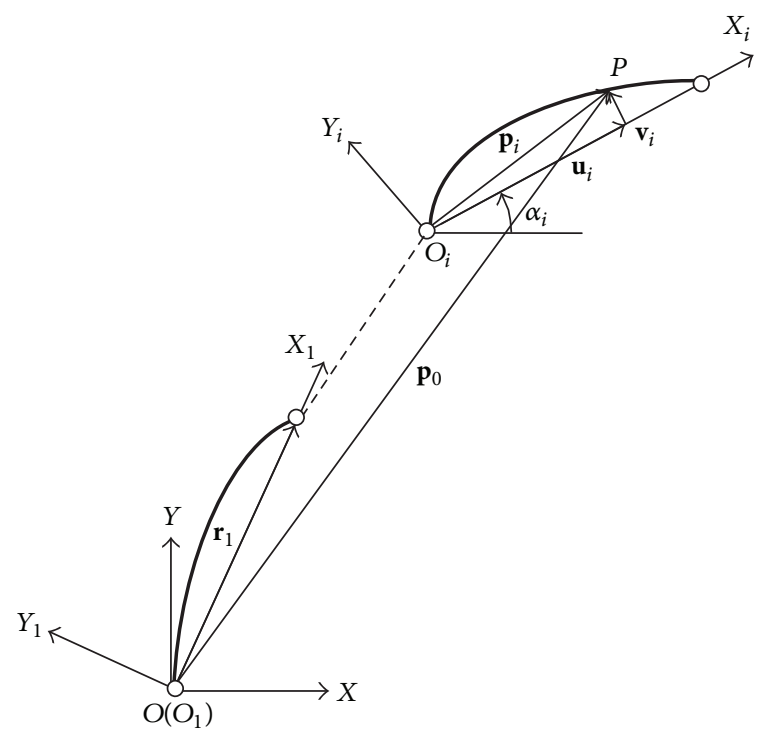

Figure 2: Model of the boom system.

in which $\mathbf{R}_{i}$ represents the orientation transformation matrix between the moving coordinate frame $O_{i} X_{i} Y_{i}$ and the inertial frame $O X Y$, and it is deduced according to the geometrical relationship presented in Figure 2:

$$
\mathbf{R}_{i}=\left[\begin{array}{cc}
\cos \alpha_{i} & -\sin \alpha_{i} \\
\sin \alpha_{i} & \cos \alpha_{i}
\end{array}\right]
$$

in which $\alpha_{i}$ represents the angle between the $X_{i}$-axis and $x$ axis.

Differentiating both sides of (1) with respect to time, the velocity of point $P$ can be obtained as follows:

$$
\dot{\mathbf{p}}_{i 0}=\sum_{k=1}^{i-1}\left(\dot{\mathbf{R}}_{k} \mathbf{r}_{k}+\mathbf{R}_{k} \dot{\mathbf{r}}_{k k+1}\right)+\dot{\mathbf{R}}_{i} \mathbf{p}_{i}+\mathbf{R}_{i} \dot{\mathbf{p}}_{i}
$$


Assuming that the booms are all homogeneous, the density of the boom can be expressed as $\rho_{i}=m_{i} / l_{i}$. Therefore, the kinetic energy of the boom system can be expressed as

$$
T=\sum_{i=1}^{5}\left(\frac{1}{2} \int_{0}^{l_{i}} \rho_{i}\left(\dot{\mathbf{p}}_{i 0}^{\mathrm{T}} \dot{\mathbf{p}}_{i 0}\right) d x_{i}\right) .
$$

Since the deformation of the booms is relatively small, the variation of the gravity potential energy caused by deformation is negligible. Thus, the potential energy of the entire system can be expressed as

$$
\begin{aligned}
U= & \sum_{i=1}^{5} \frac{1}{2} E I_{i} \int_{0}^{l_{i}}\left(\frac{\partial^{2} v_{i}}{\partial x_{i}^{2}}\right)^{2} d x_{i} \\
& +\sum_{i=1}^{5} m_{i} g\left(\frac{l_{i}}{2} \sin \alpha_{i}+\sum_{k=1}^{i-1} l_{k} \sin \alpha_{k}\right),
\end{aligned}
$$

in which $E$ represents the elasticity modulus of the material and $I_{i}$ represents the second moment of area of the $i$ th boom.

According to the assumed mode method, the deformation $v_{i}$ can be obtained by utilizing the first second order Ritz functions, which generally satisfy the accuracy requirement.

The generalized coordinates vector can be defined as $\mathbf{q}=$ $\left[\begin{array}{ll}\boldsymbol{\theta} & \mathbf{d}\end{array}\right]^{\mathrm{T}}$, in which $\boldsymbol{\theta}$ represents the joint angle vector and $\mathbf{d}$ represents the deformation vector. According to the Lagrange equations of the second kind, the flexible multibody dynamic equation of the boom system can be deduced as

$$
M \ddot{q}+C \dot{q}+K q=T F,
$$

in which $\mathbf{M}, \mathbf{C}$, and $\mathbf{K}$ represent the inertial, damping, and stiffness matrices of the system, respectively. $\mathbf{F}$ represents the control force vector acting on the boom system, and $\mathbf{T}$ represents the kinematic relationship matrix.

According to (6), the undamped free vibration equation of the system can be deduced:

$$
\mathbf{M} \ddot{\mathbf{q}}+\mathbf{K q}=\mathbf{0} .
$$

Modal analysis of the boom system is implemented for the horizontal configuration, since the damage caused by vibration is more serious in this case. Table 1 shows the natural frequencies of the first three modes obtained by numerical simulation. In addition, the experimental frequency response of the boom system is shown in Figure 3 for comparison. It can be learned that the first two natural frequencies acquired experimentally can accord with the numerical results, which validates the established system model. In practical application, the pumping frequency is generally limited between $0.2167 \mathrm{~Hz}$ and $0.3667 \mathrm{~Hz}$, and it is close to the first order natural frequency of the boom system, which easily leads to resonance.

Figure 4 shows the first two modes of vibration of the boom system, respectively, which are obtained by numerical analysis. Table 2 gives the corresponding vibration state of each boom in these two modes. It can be realized that vibration of the first three booms is very weak and can be negligible comparing with the other booms, due to their higher stiffness.
TABLE 1: The natural frequencies of the boom system.

\begin{tabular}{lccc}
\hline Natural frequency & 1st & 2nd & 3rd \\
\hline Value $(\mathrm{Hz})$ & 0.3807 & 0.9053 & 1.6799 \\
\hline
\end{tabular}

TABle 2: The maximum modal displacement of each boom in the first two modes.

\begin{tabular}{lcc}
\hline The number of the boom & The first mode & The second mode \\
\hline The first boom & $1 \times 10^{-4}$ & $3 \times 10^{-4}$ \\
The second boom & $3 \times 10^{-4}$ & $15 \times 10^{-4}$ \\
The third boom & $16 \times 10^{-4}$ & $7 \times 10^{-3}$ \\
The fourth boom & $28 \times 10^{-2}$ & $9 \times 10^{-1}$ \\
The fifth boom & $1 \times 10^{0}$ & $1 \times 10^{0}$ \\
\hline
\end{tabular}

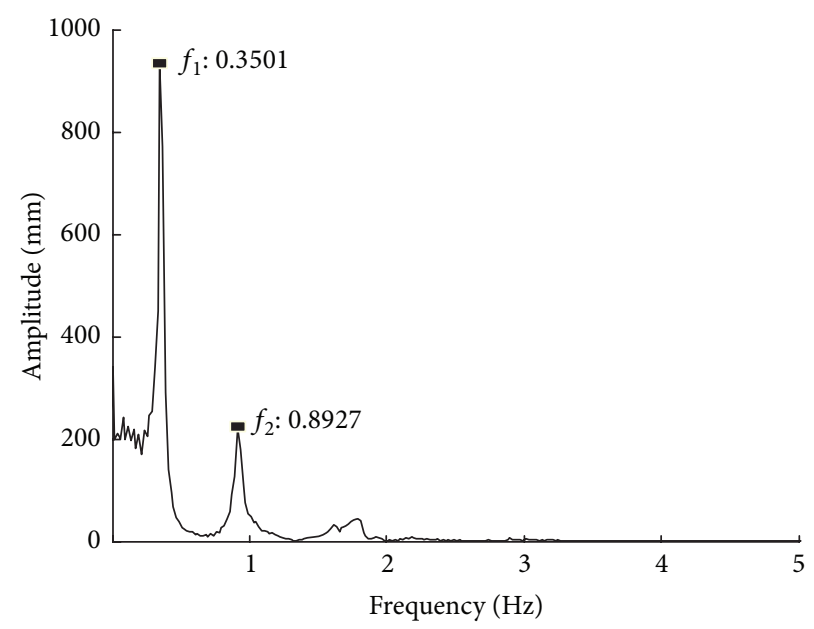

FIGURE 3: Experimental frequency response of the boom system.

\section{Vibration Control of the Boom System}

This section presents an active control strategy for suppressing vibration. The control strategy proposed is based on independent modal approach. Based on a set of independent modal equations, the control law containing two impulses is designed to suppress vibration according to the principle of original CIST. Moreover, the deviation of the equilibrium position after implementing control action is also taken into account. And the hydraulic cylinders used for driving the booms are considered as the actuators to suppress vibration in this work, without introducing other actuators. The modal coordinates used to describe vibratory state of the boom system cannot be measured directly, and they are estimated through a modal state observer.

3.1. Principle of CIST. CIST is a new control strategy for suppressing the vibration induced by external disturbance, which is an evolution of IST. However, its principle is different from the well-known time-lag filtering of IST. Actually, the basic idea of CIST is to generate the appropriate impulse which triggers the reverse vibration to counteract the original one, so that the original vibration can be completely eliminated. The principle of CIST is shown in Figure 5; when vibration 


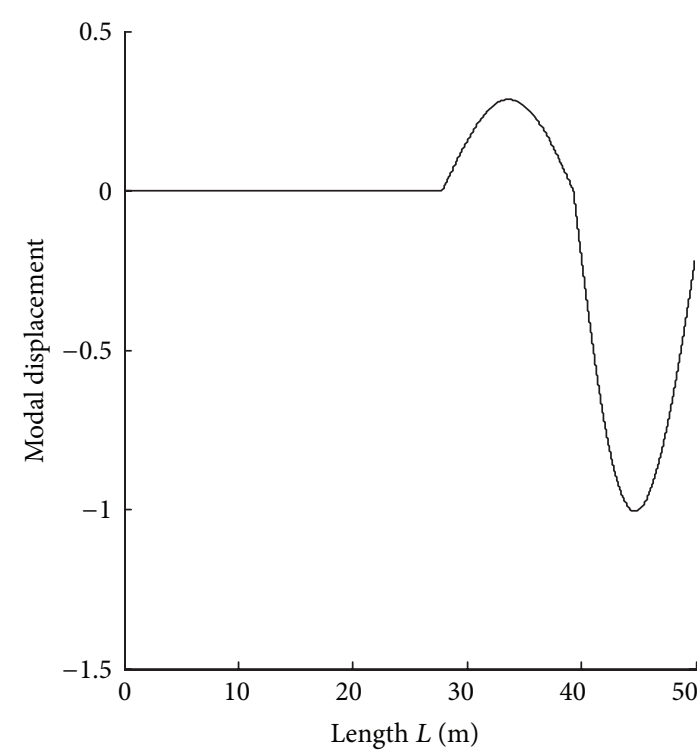

(a) The first mode

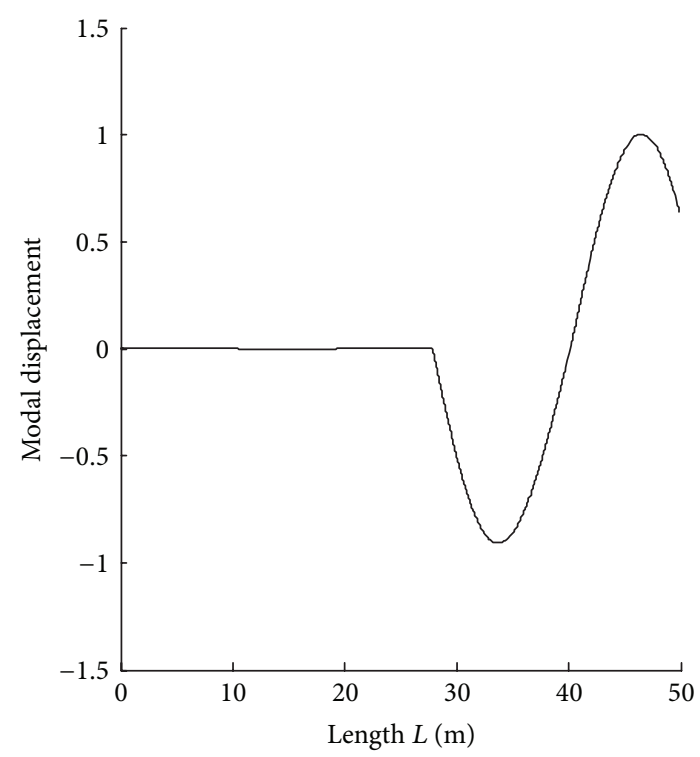

(b) The second mode

FIGURE 4: Modal of vibration of the boom system: (a) the first mode, (b) the second mode.

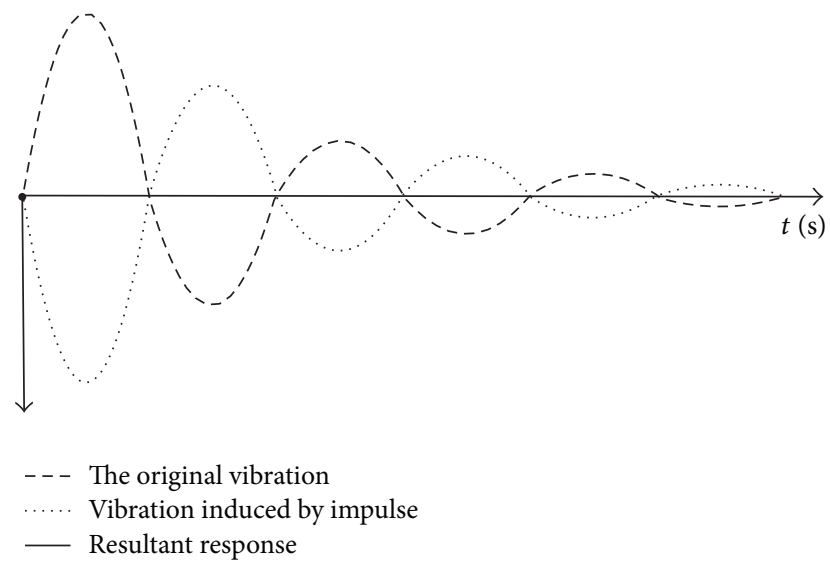

FIGURE 5: The principle of CIST.

of the flexible structure is detected, an impulse generated by CIST will be imposed on the flexible system at once. Then, vibration is eliminated through the superposition of impulse responses.

3.2. Independent Modal Approach. Generally, not all the modes should be considered in vibration control because only the former modes play an important part in dynamics of the boom system. Therefore, only the former modes are considered in this work. Defining $n$ as the number of modes considered, the eigenvector matrix of the considered modes can be expressed as

$$
\Phi=\left[\begin{array}{lll}
\phi_{1} & \cdots & \phi_{n}
\end{array}\right]
$$

in which $\phi_{j}$ represents the eigenvector of the $j$ th mode considered.
To apply the independent modal approach, the equation needs to be decoupled by defining the transformation in modal coordinates, which is expressed as

$$
\mathbf{q}=\Phi \eta
$$

in which $\boldsymbol{\eta}$ represents the vector of modal coordinates.

Substituting (9) into (6) and left multiplying both sides of the new expression of (6) with $\boldsymbol{\Phi}^{\mathrm{T}}$ yield

$$
\boldsymbol{\Phi}^{\mathrm{T}} \mathbf{M} \boldsymbol{\Phi} \ddot{\boldsymbol{\eta}}+\boldsymbol{\Phi}^{\mathrm{T}} \mathbf{C} \boldsymbol{\Phi} \dot{\boldsymbol{\eta}}+\boldsymbol{\Phi}^{\mathrm{T}} \mathbf{K} \boldsymbol{\Phi} \boldsymbol{\eta}=\boldsymbol{\Phi}^{\mathrm{T}} \mathbf{T F} .
$$

The damping matrix in equation is defined according to the Rayleigh damping assumption:

$$
\mathbf{C}=a \mathbf{M}+b \mathbf{K},
$$

in which the coefficients $a$ and $b$ are obtained experimentally.

Therefore, the decoupled equation can be deduced as

$$
\widehat{\mathbf{M}} \ddot{\boldsymbol{\eta}}+\widehat{\mathbf{C}} \dot{\boldsymbol{\eta}}+\widehat{\mathbf{K}} \boldsymbol{\eta}=\mathbf{U}_{\mathrm{C}},
$$

in which $\widehat{\mathbf{M}}=\boldsymbol{\Phi}^{\mathrm{T}} \mathbf{M} \boldsymbol{\Phi}, \widehat{\mathbf{C}}=\boldsymbol{\Phi}^{\mathrm{T}} \mathbf{C} \boldsymbol{\Phi}, \widehat{\mathbf{K}}=\boldsymbol{\Phi}^{\mathrm{T}} \mathbf{K} \boldsymbol{\Phi}, \mathbf{U}_{C}=\boldsymbol{\Phi}^{\mathrm{T}} \mathbf{T F}$, and $\widehat{\mathbf{M}}, \widehat{\mathbf{C}}$, and $\widehat{\mathbf{K}}$ are the diagonal matrices. Therefore, a set of independent modal equations are obtained according to (12):

$$
m_{j} \ddot{\eta}_{j}+c_{j} \dot{\eta}_{j}+k_{j} \eta_{j}=u_{j} .
$$

3.3. Vibration Control Based on Constant-Position CIST. According to the principle of CIST in Figure 5, the vibration of the boom system can be eliminated completely by applying an appropriate impulse at the very moment of vibration occurrence in theory. However, in view of the characteristics of time-lag existing in the real boom system and variation of the equilibrium position after implementing the control action, the original control strategy will become insufficient 


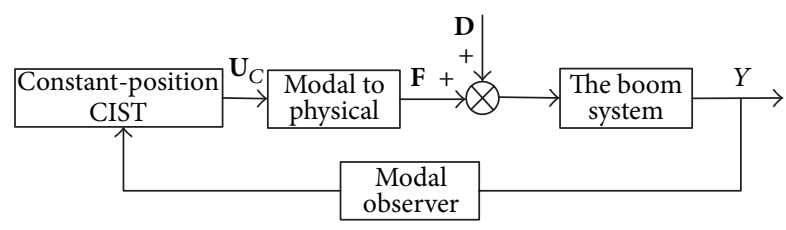

FIGURE 6: Block diagram of vibration control.

in practical application. To achieve the preferable control performance, constant-position CIST is proposed in this work, and the control block diagram is shown in Figure 6, in which $D$ represents the external disturbance.

In this work, the first two modes are considered for vibration control, and the corresponding actuators are required to implement control action. Because of weak vibration of the first three booms according to the numerical results described in Section 2, the last two actuators are considered for implementing control action. According to the acquired independent modal equations, the transfer function can be described by the second order system:

$$
G_{j}(s)=\frac{\omega_{j}^{2}}{s^{2}+2 \xi_{j} \omega_{j} s+\omega_{j}^{2}},
$$

in which $\xi_{j}$ and $\omega_{j}$ represent the corresponding damping ratio and natural frequency, respectively.

According to [17], the response of single impulse disturbance can be expressed as

$$
\eta_{j d}(s)=\frac{s \eta_{j 0}+2 \xi_{j} \omega_{j} \eta_{j 0}+\dot{\eta}_{j 0}}{s^{2}+2 \xi_{j} \omega_{j} s+\omega_{j}^{2}},
$$

in which $\eta_{j 0}$ and $\dot{\eta}_{j 0}$ represent the initial modal coordinate and velocity, respectively.

In this section, double-impulse control with reverse direction is designed for the considered modes, which is presented by (16). The principle of double-impulse control is described in Figure 7. According to this figure, it can be learned that the original vibration is eliminated by the superposition of two impulses responses; furthermore, the designed two impulses with reverse direction are also beneficial to avoid the boom deviating from the original equilibrium position in control process because the variation of equilibrium position caused by one impulse can be counteracted by the other:

$$
u_{j}(s)=u_{j 1} e^{-s t_{j 1}}+u_{j 2} e^{-s t_{j 2}},
$$

in which $u_{j 1}$ and $u_{j 2}$ denote the amplitudes of the two impulses and $t_{j 1}$ and $t_{j 2}$ denote the acting moments of these two impulses, respectively.

Taking the time-lag characteristic of the hydraulic system into account, the dynamics of single hydraulic driving system can be approximately expressed as follows with respect to impulse action:

$$
G_{h}(s)=K e^{-s t_{h}},
$$

in which $K$ denotes the proportional gain and $t_{h}$ represents the lagging time. These parameters are all estimated according to experimental data.

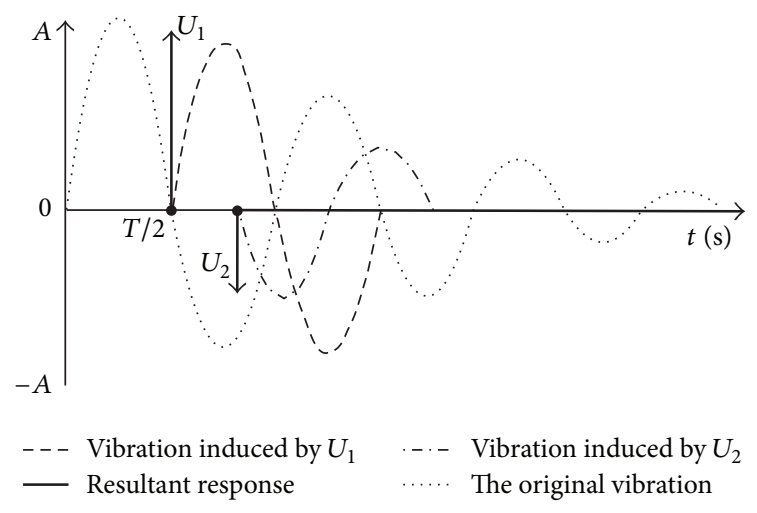

FIGURE 7: The principle of double-impulse control.

According to the designed control law, the active control response can be obtained as

$$
\eta_{c j}(s)=\frac{K e^{-s t_{h}}\left(u_{j 1} e^{-s t_{j 1}}+u_{j 2} e^{-s t_{j 2}}\right) \omega_{j}^{2}}{s^{2}+2 \xi_{j} \omega_{j} s+\omega_{j}^{2}} .
$$

In practice, the action of the first impulse is not simultaneous with the occurrence of the vibration due to the existing lagging time. In this work, the first impulse is designed to take effect at the very moment of half-vibration period after vibration occurs. Moreover, the lagging time has also been taken into account to avoid its adverse effect. Therefore, $t_{j 1}$ can be determined by

$$
t_{j 1}=\frac{T_{j}}{2}-t_{h}
$$

in which $T_{j}$ represents the corresponding vibration period.

According to the transformation in aforementioned modal coordinates, the practical control action directly depending on the modal coordinates can be deduced as

$$
\mathbf{F}=\left(\boldsymbol{\Phi}^{\mathrm{T}} \mathbf{T}\right)^{-1} \mathbf{U}_{\mathrm{C}} .
$$

Defining $\mathbf{D}=\left(\boldsymbol{\Phi}^{\mathrm{T}} \mathbf{T}\right)^{-1}$, because the number of control forces equals the number of considered modes, $\mathbf{D}$ is an $n \times n$ matrix. The $m$ th element in vector $\mathbf{F}$ can be described as

$$
f_{m}=\sum_{j=1}^{n} d_{m j} u_{j},
$$

in which $d_{m j}$ represents the element of $\mathbf{D}$ and the subscript $m$ represents an integer between 1 and $n$.

According to the proposed control law, $u_{j}$ is composed of two impulses. And the impulses are specially designed to satisfy an appropriate relationship in this work, following which the variations of the equilibrium position caused by each impulse can be mutually counteracted, so that final variation of the equilibrium position is avoided after control action is implemented. The relationship should be deduced according to the hydraulic system. As a result, from the equilibrium 
equation with respect to the $m$ th actuator, it can be deduced that

$$
\begin{aligned}
\frac{\int\left(K_{q 1} k d_{m j} u_{j 1}-Q_{m 1}\right) \mathbf{d} t}{A_{m 1}} & \\
+\frac{\int\left(K_{q 2} k d_{m j} u_{j 2}-Q_{m 2}\right) \mathbf{d} t}{A_{m 2}} & =0,
\end{aligned}
$$

in which $A_{m 1}$ and $A_{m 2}$ represent the effective areas of the two chambers of the actuator, $Q_{m 1}$ and $Q_{m 2}$ represent the total leakage in each side, $K_{q 1}$ and $K_{q 2}$ represent flow coefficients in corresponding workstations of the corresponding valve, and $k$ represents the amplification gain of the valve.

Because the action time of each impulse is the same as the others in practice, the relationship can be deduced according to $(22)$ :

$$
u_{j 1}=\frac{A_{m 1}\left(K_{q 2} k d_{m j} u_{j 2}-Q_{m 2}\right)+A_{m 2} Q_{m 1}}{A_{m 2} K_{q 1} k d_{m j}} .
$$

According to the principle of CIST illustrated in Section 3.1, and the relationship between the two impulses, control action $u_{j}$ with respect to the $m$ th actuator can be obtained, and the corresponding parameters are solved as

$$
\begin{aligned}
t_{j 1}= & \frac{T_{j}}{2}-t_{h}, \\
t_{j 2} & \\
= & \frac{\left(\sin ^{-1}\left(K_{q 2} A_{m 1} N /\left(M^{2}+\left(N K_{q 1} A_{m 2}\right)^{2}\right)\right)-\varphi\right)}{\omega_{d j}} \\
& -t_{h}, \\
u_{j 1} & =\frac{\sigma \eta_{j 0}+\dot{\eta}_{j 0}+\eta_{j 0} \omega_{d j} \cot \left[\omega_{d j}\left(t_{j 2}+t_{h}\right)\right]}{K \omega_{j}^{2}}, \\
u_{j 2} & =\frac{\eta_{j 0} \omega_{d j}}{K \omega_{j}^{2} \sin \left[\omega_{d j}\left(t_{j 2}+t_{h}\right)\right]},
\end{aligned}
$$

in which $\varphi=\tan ^{-1}\left(N K_{q 1} A_{m 2} / M\right), N=\eta_{j 0} \omega_{d j} k d_{m j}, M=$ $K_{q 1} A_{m 2} k d_{m j}\left(\sigma \eta_{j 0}+\dot{\eta}_{j 0}\right)+K \omega_{j}^{2}\left(A_{m 1} Q_{m 2}-A_{m 2} Q_{m 1}\right), \sigma=\xi_{j} \omega_{j}$, and $\omega_{d j}=\omega_{j} \sqrt{1-\xi_{j}^{2}}$.

3.4. Modal Coordinates Estimation. The proposed control strategy is based on independent modal approach. Modal coordinates of the considered modes are necessary for control action, which affect the final control performance. Generally, the modal coordinates cannot be measured directly, which need to be estimated by a modal observer, which is presented in this section. to $(6)$ :

The state equation of the system can be defined according

$$
\begin{aligned}
& \dot{\mathbf{X}}=\mathbf{A X}+\mathbf{B F}, \\
& \mathbf{Y}=\mathbf{P X}
\end{aligned}
$$

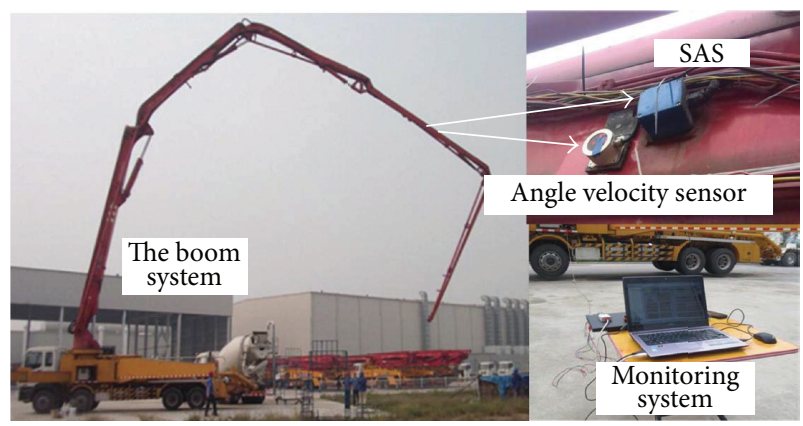

Figure 8: The experimental set.

in which $\mathbf{X}$ represents the state-space vector, $\mathbf{Y}$ represents the measurements vector, and $\mathbf{P}$ represents the relationship between the measurements and the system coordinates.

Because the modal coordinates are required in this work, it is necessary to switch to modal components. Similarly, the transformation can be defined as

$$
\mathrm{X}=\boldsymbol{\Phi} \boldsymbol{\eta}
$$

Similarly, substituting (26) to (25) and left multiplying both sides of the generated equation with $\Phi^{-1}$ yield

$$
\begin{aligned}
& \dot{\boldsymbol{\eta}}=\mathbf{A}_{k} \boldsymbol{\eta}+\mathbf{B}_{k} \mathbf{F} \\
& \mathbf{Y}=\mathbf{P}_{k} \boldsymbol{\eta}
\end{aligned}
$$

in which $\mathbf{A}_{k}=\Phi^{-1} \mathbf{A} \Phi, \mathbf{B}_{k}=\Phi^{-1} \mathbf{B} \Phi$, and $\mathbf{P}_{k}=\mathbf{P} \Phi$.

The modal observer is defined as

$$
\begin{aligned}
& \dot{\hat{\boldsymbol{\eta}}}=\mathbf{A}_{k} \widehat{\boldsymbol{\eta}}+\mathbf{B}_{k} \mathbf{F}+\mathbf{H}(\mathbf{Y}-\widehat{\mathbf{Y}}), \\
& \widehat{\mathbf{Y}}=\mathbf{P}_{k} \widehat{\boldsymbol{\eta}},
\end{aligned}
$$

in which $\widehat{\boldsymbol{\eta}}$ represents the estimated vector of modal coordinates, $\widehat{\mathbf{Y}}$ represents estimated measurements vector, and $\mathbf{H}$ represents the gain matrix of the observer, which is calculated by adopting the well-known poles placement method.

\section{Experiments and Analysis}

To verify the effectiveness of the proposed control strategy, experiments of vibration control were implemented on a real 52-meter-long five-boom system shown in Figure 8. The original 16-bit PLC was preserved as the core controller to implement active control without assistance from other equipment, which is fit for the practical engineering application. The boom state was detected by space angle sensor (SAS) and angular velocity sensor shown in Figure 8. Impulse is not possible to be implemented in practice, in view of which, pulse was applied for implementation to replace impulse.

External disturbances imposed on the boom system are composed of a lot of consecutive impulses. Vibration control experiments were primarily implemented to deal with single impulse disturbance. At the beginning, the boom system was approximately extended to the horizontal configuration for experiment because larger vibration will be excited obviously 


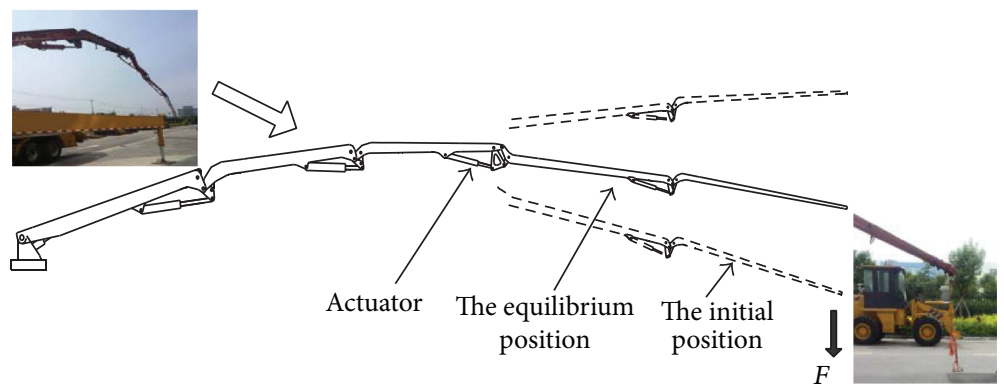

FIGURE 9: Principle of vibration exciting.
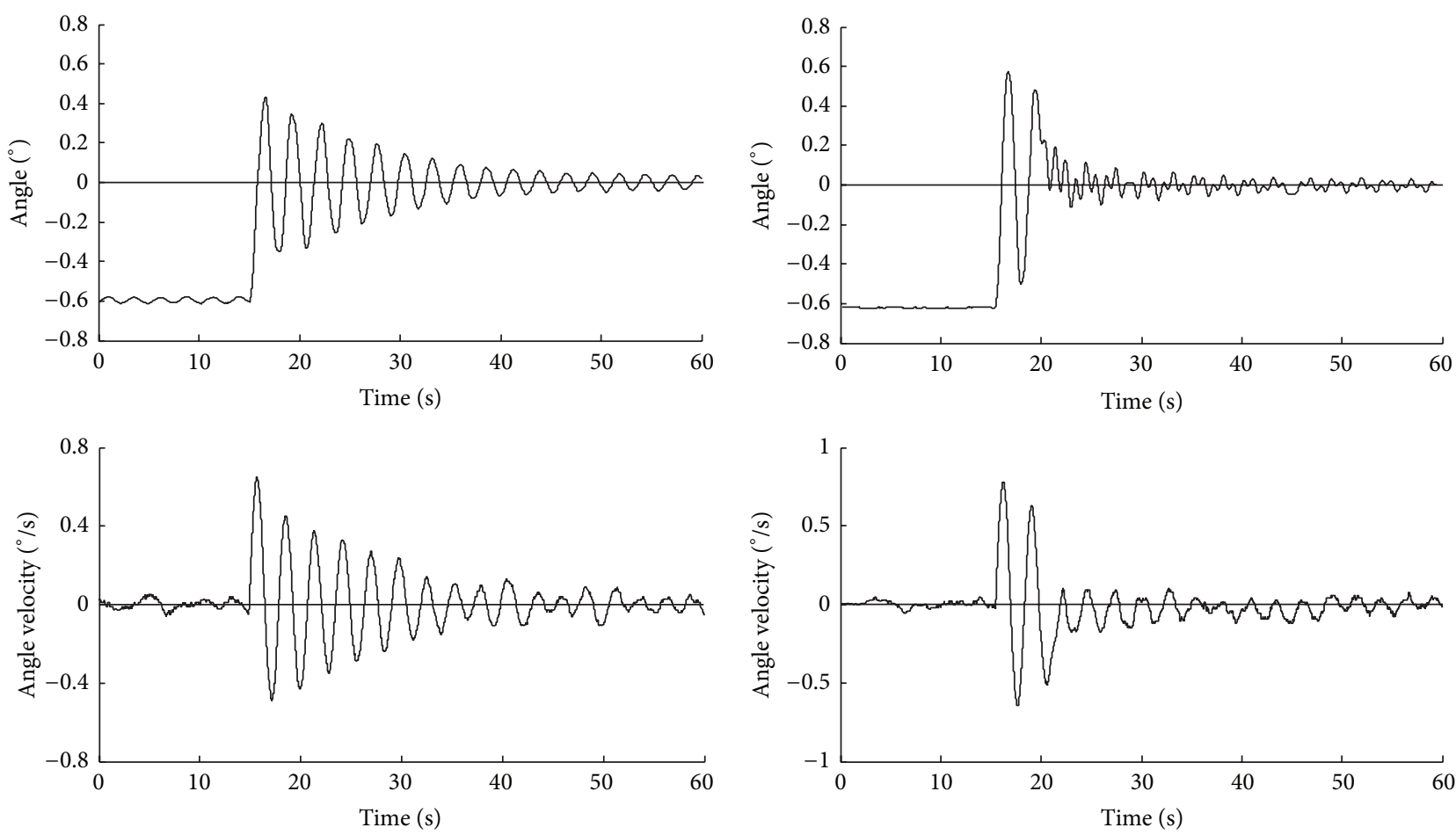

(a) Free vibration

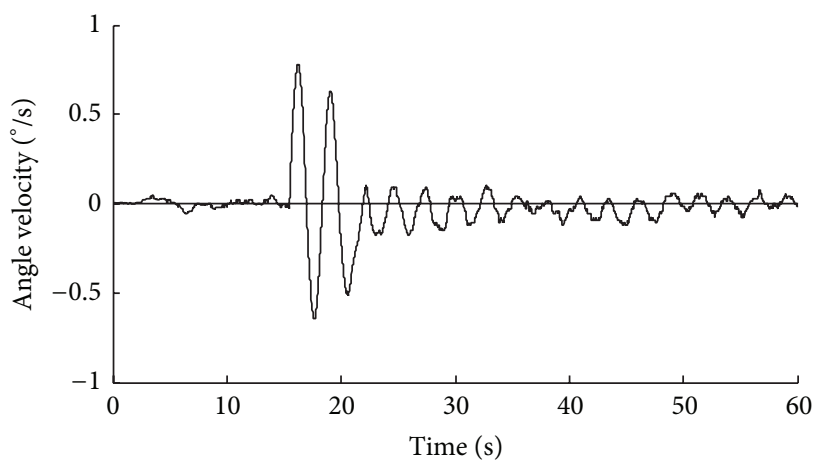

(b) Vibration control

FIGURE 10: Experimental results of the fourth boom under single impulse disturbance.

in this case. The original vibration was produced by means of external equipment. Initially, the boom system was deviated from the equilibrium position by external force, and vibration was excited at the moment of repealing external force. Figure 9 shows the principle of exciting vibration, and Figure 10 presents the experimental results by adopting constant-position CIST, in which the initial equilibrium position is marked as zero. In order to verify the effectiveness of the proposed control strategy, free vibration of the boom system is also presented for comparison. According to the experimental results, it can be learned that the vibration is reduced about $65 \%$ by adopting the active control action, and the maximum angular velocity is also reduced from $0.87 \%$ s to $0.16 \%$ s. Therefore, it can be concluded that the proposed control strategy is effective to suppress the vibration which is caused by single impulse disturbance. In addition, when the boom tends towards stability, the new equilibrium position does not deviate from the initial one, which states that constantposition CIST is not only effective to accomplish vibration suppression, but also able to avoid the variation of the equilibrium position after implementing the control action.

Considering periodic shocks inevitably disturbing the boom system, constant-position CIST is also required to counteract the periodic external disturbances in practice. In this section, pumping concrete is replaced by pumping water to produce vibration. Experimental results with respect to periodic disturbances are represented in Figure 11. In the beginning of this experiment, vibration was excited and lasted. Then, the control action was started at 42 seconds. To compare the vibration state with and without active control 


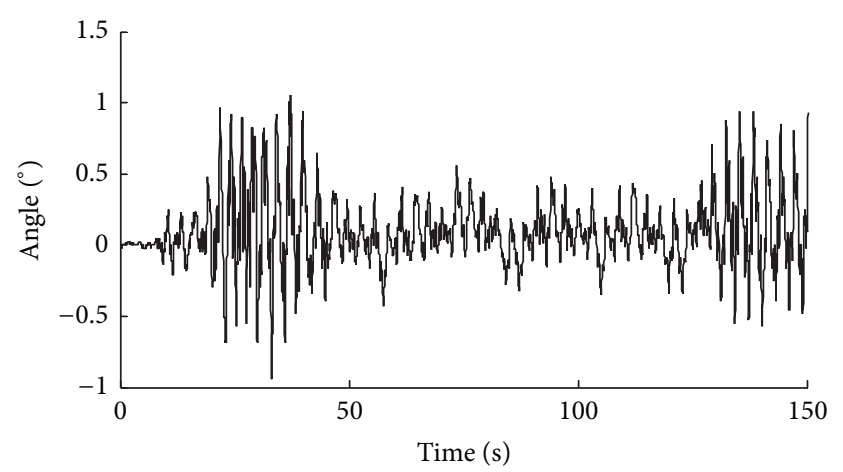

(a) The vibratory state

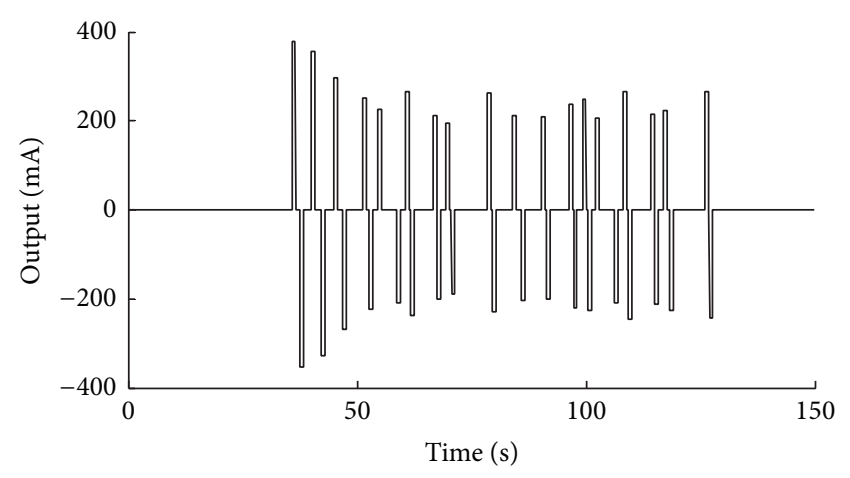

(b) The corresponding control action

FIGURE 11: Experimental results of the fourth boom with and without constant-position CIST under periodic disturbances.

action, the control action was repealed at 127 seconds. According to the experimental results, it is seen that vibration is reduced about $55 \%$ by the effect of the initial control action, and it is limited in a small range later due to the subsequent control action. Moreover, little deviation of the equilibrium position has been detected in process. Figure 11 also presents the corresponding control action relation to the fourth actuator. According to the figure, it is learned that positive action and negative action are generated alternatively, which accords with the proposed control strategy. In addition, the control action can also adapt to the real vibratory state of the system. It can be obviously acquired that constant-position is also effective for periodic disturbances. The range is determined by the given threshold, but its value cannot be set too small; otherwise system stability may be deteriorated as weak vibration is excited.

\section{Conclusions}

For the vibration of the boom system caused by pumping concrete in construction, the active control strategy of constantposition CIST is discussed in this work.

According to a set of the independent modal equations deduced by independent modal approach and the considered control modes, the control action consisting of two impulses with reverse direction is designed to suppress vibration and avoid the variation of the equilibrium position at the end. In addition, the lagging time existing in practical application is also taken into account for obtaining preferable control action.

Experiments of suppressing the vibration caused by single disturbance and periodic disturbances were implemented, respectively. The results show that the vibration can be reduced obviously in a short time by adopting the active control strategy. In addition, the variation of the equilibrium position can hardly be detected according to the experimental results.

These promising results indicate that the proposed control strategy is able to overcome the external disturbances induced by pumping concrete and satisfies the requirement of vibration control in practical application.

\section{Conflict of Interests}

The authors declare that there is no conflict of interests regarding the publication of this paper.

\section{Acknowledgment}

This work is supported by Jiangsu Xuzhou Construction Machinery Research Institute in China.

\section{References}

[1] X. Sun, H. Ye, and S. Fei, "A closed-loop detection and openloop control strategy for booms of truck-mounted concrete pump," Automation in Construction, vol. 31, pp. 265-273, 2013.

[2] G. Cazzulani, C. Ghielmetti, H. Giberti, F. Resta, and F. Ripamonti, "Overview on the truck mounted concrete boom pump: a dynamic numerical model for active control logic definition," in Proceedings of the 18th IFAC World Congress, pp. 4232-4237, Milano, Italy, August 2011.

[3] G. Cazzulani, C. Ghielmetti, H. Giberti, F. Resta, and F. Ripamonti, "A test rig and numerical model for investigating truckmounted concrete pumps," Automation in Construction, vol. 20, no. 8, pp. 1133-1142, 2011.

[4] F. Resta, F. Ripamonti, G. Cazzulani, and M. Ferrari, "Independent modal control for nonlinear flexible structures: an experimental test rig," Journal of Sound and Vibration, vol. 329, no. 8, pp. 961-972, 2010.

[5] G. Cazzulani, F. Resta, and F. Ripamonti, "A feedback and feedforward vibration control for a concrete placing boom," Journal of Vibration and Acoustics, vol. 133, no. 5, Article ID 051002, pp. 1-8, 2011.

[6] G. Bagordo, G. Cazzulani, F. Resta, and F. Ripamonti, "A modal disturbance estimator for vibration suppression in nonlinear flexible structures," Journal of Sound and Vibration, vol. 330, no. 25, pp. 6061-6069, 2011.

[7] Y. Huang, B.-X. Wu, and J.-Q. Wang, "Test for active control of boom vibration of a concrete pump truck," Journal of Vibration and Shock, vol. 31, no. 2, pp. 91-94, 2012.

[8] Z. Y. Wu, X. Zhou, D. Hu, and X. Kang, "Active vibration suppression for boom of concrete pump truck based on timedelay compensation approach," China Mechanical Engineering, vol. 24 , no. 24 , pp. 3283-3288, 2013. 
[9] N. C. Singer and W. P. Seering, "Preshaping command inputs to reduce system vibration," Journal of Dynamic Systems, Measurement and Control, vol. 112, no. 1, pp. 76-82, 1990.

[10] N. C. Singer and W. P. Seering, "An extension of command shaping methods for controlling residual vibration using frequency sampling," in Proceedings of the IEEE International Conference on Robotics and Automation, vol. 1, pp. 800-805, IEEE, Nice, France, May 1992.

[11] J. Park and P. H. Chang, "Use of input shaping technique with a robust feedback control and its application to the position control of surface mount machine," in Proceedings of the IEEE International Conference on Control Applications, pp. 397-402, Dearborn, Mich, USA, September 1996.

[12] W. E. Singhose, L. J. Porter, and W. P. Seering, "Input shaped control of a planar gantry crane with hoisting," in Proceedings of the American Control Conference, pp. 97-100, Albuquerque, NM, USA, June 1997.

[13] M. Bodson, "An adaptive algorithm for the tuning of two input shaping methods," Automatica, vol. 34, no. 6, pp. 771-776, 1998.

[14] C. F. Cutforth and L. Y. Pao, "Adaptive input shaping for maneuvering flexible structures," Automatica, vol. 40, no. 4, pp. 685693, 2004.

[15] W. E. Singhose, W. P. Seering, and N. C. Singer, "Time-optimal negative input shapers," ASME Journal of Dynamic Systems, Measurement and Control, vol. 119, no. 2, pp. 198-205, 1997.

[16] L. Y. Pao and M. A. Lau, "Robust input shaper control design for parameter variations in flexible structures," Journal of Dynamic Systems Measurement and Control, vol. 122, no. 1, pp. 63-70, 2000.

[17] P. H. Chang, J. Park, and J. Y. Park, "Commandless input shaping technique," in Proceedings of the American Control Conference, pp. 299-304, Arlington, Tex, USA, June 2001.

[18] J.-Y. Park and P.-H. Chang, "Vibration control of a telescopic handler using time delay control and commandless input shaping technique," Control Engineering Practice, vol. 12, no. 6, pp. 769-780, 2004. 

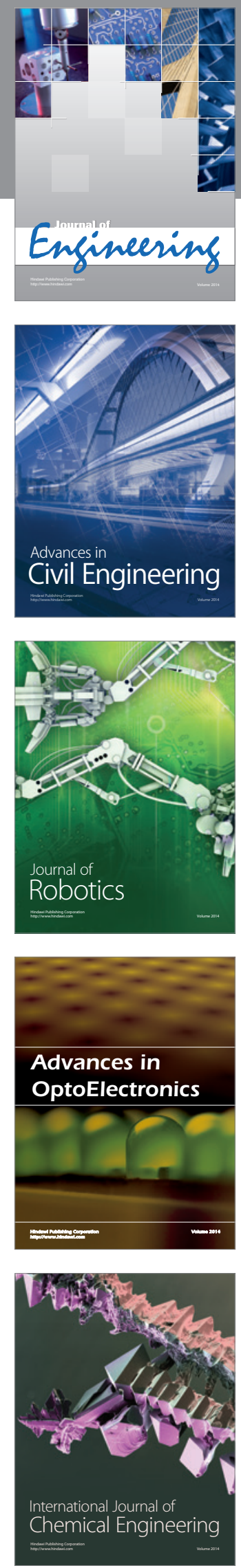

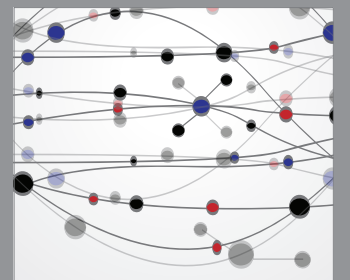

The Scientific World Journal
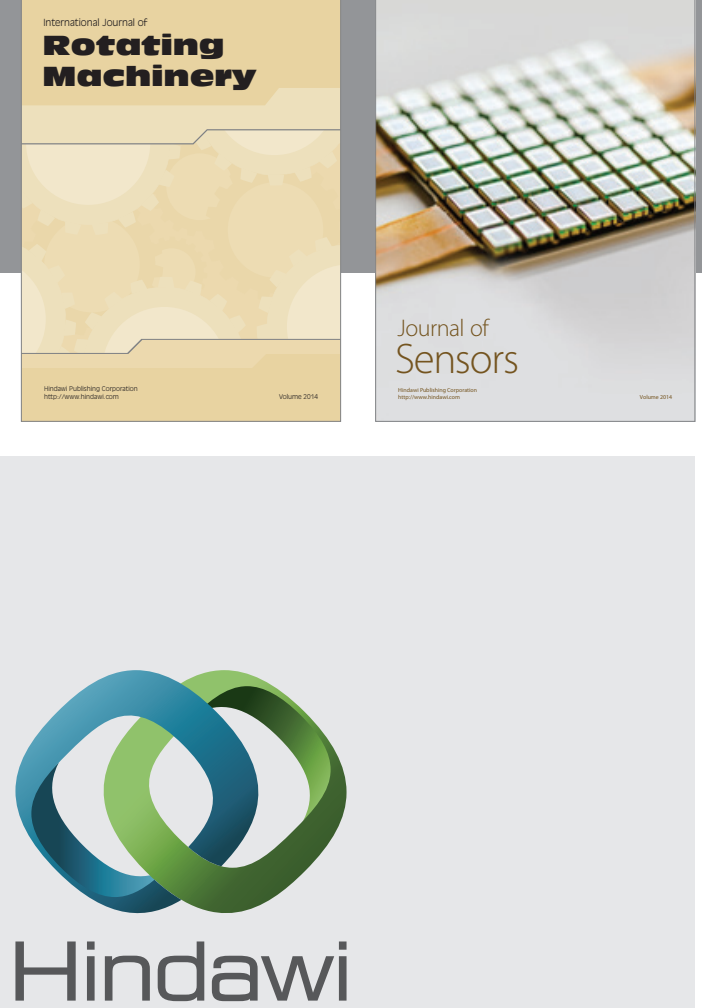

Submit your manuscripts at http://www.hindawi.com
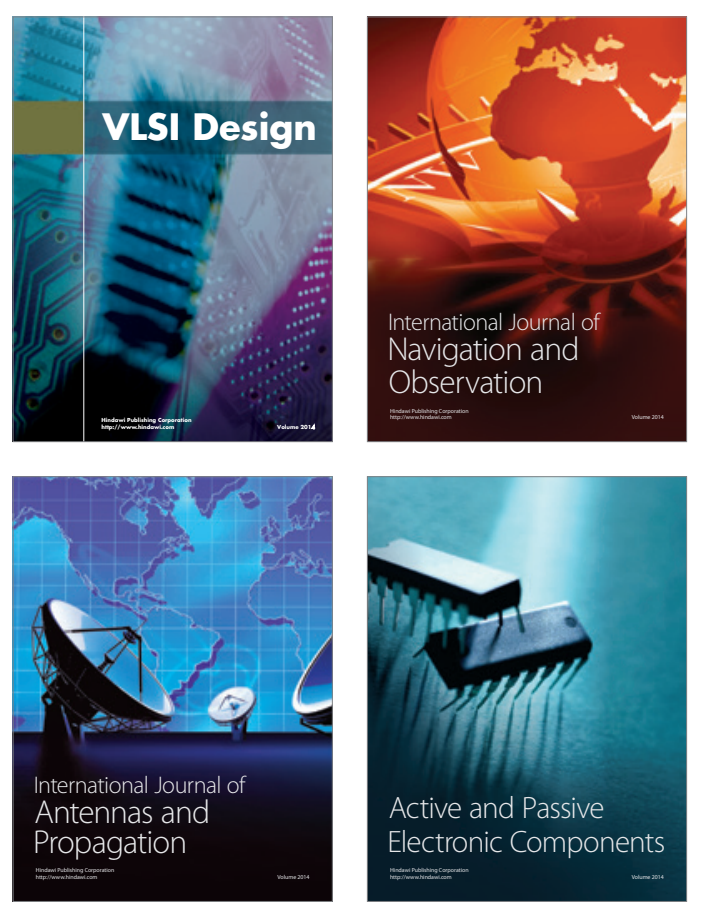
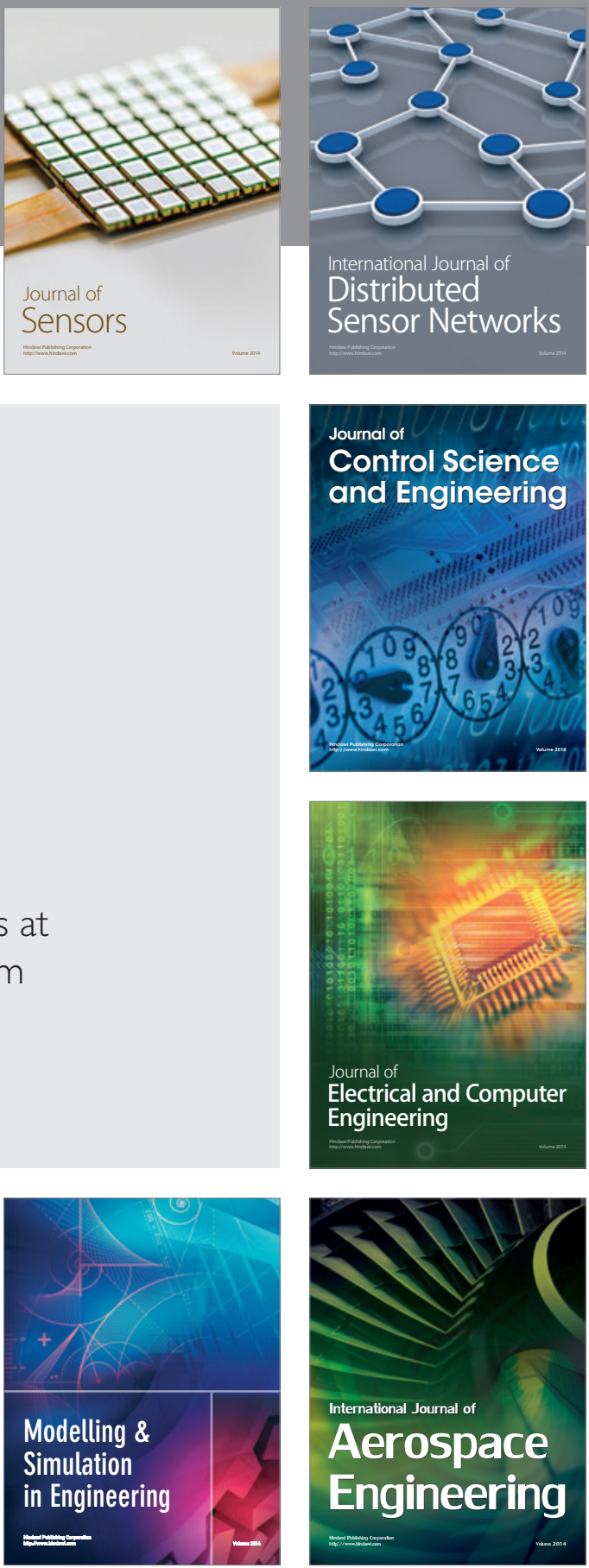

Journal of

Control Science

and Engineering
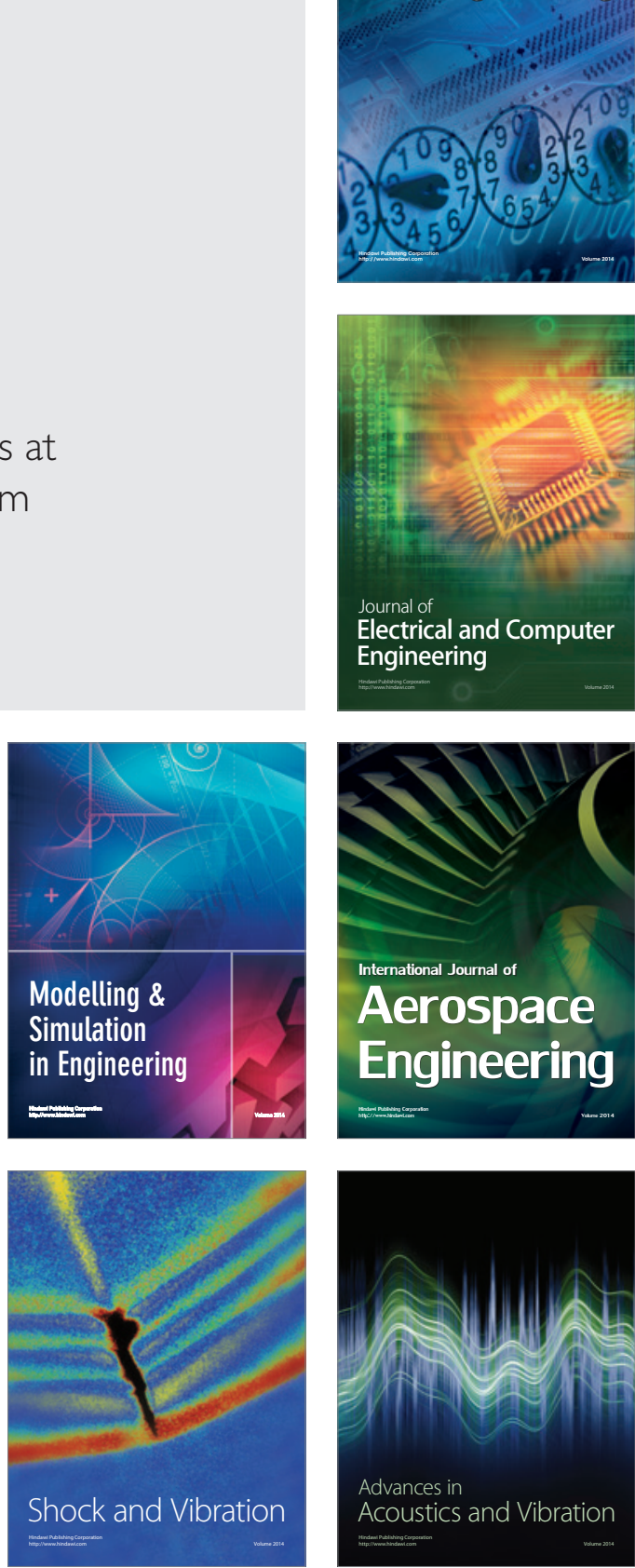